\title{
Integrative Arbeit mit dem Psychodrama
}

\author{
Falko von Ameln • Stefan Gunkel
}

Online publiziert: 17. September 2014

(C) Springer Fachmedien Wiesbaden 2014

Wenngleich davon auszugehen ist, dass viele PsychodramatikerInnen kein "schulenreines" Psychodrama einsetzen, sondern auch Elemente aus anderen Verfahren in ihre Arbeit integrieren, sind mögliche Perspektiven einer integrativen Arbeit mit dem Psychodrama in der Literatur nur punktuell beschrieben. Dies mag auf Konkurrenzerleben und Abgrenzungsbestrebungen gegenüber einer als übermächtig erlebten Verhaltenstherapie, auf den Wunsch nach einer klaren Identität oder der hohen Identifikation mit Morenos Methoden- und Theoriekosmos zurückgehen. Wenngleich das psychodramatische Verfahren mit seinen Theoriebeständen zweifellos ein hohes und bislang außerhalb der Community weithin unerkanntes Potenzial für eine komplexe Sicht auf den Menschen bietet (vgl. das Interview mit Hilarion Petzold zu Beginn dieses Bandes sowie den kürzlich erschienenen ZPS-Sonderband „Jacob Levy Moreno revisited“", Ameln und Wieser 2014), droht eine selbstreferenzielle Beschränkung auf ein tradiertes und als ,rein“ verstandenes Psychodrama in eine Wagenburg-Mentalität und letztlich in traditionalistische Erstarrung zu münden:

„The presumption among psychodramatists has been that all the ideas for innovations lie within Moreno's theoretical ideas [...]. Traditional psychodramatists spend their time trying to fit psychodramatic concepts to other theories rather than transporting external concepts that can enrich the practice of psychodrama and improve on its classical methodology [...]. For all its insightful ideas,

\footnotetext{
Dipl.-Psych. PP S. Gunkel ( $₫)$

KRH - Klinik für Psychiatrie und Psychotherapie,

Psychologischer Psychotherapeut,

Rohdehof 3, 30853 Langenhagen, Deutschland

E-Mail: stefan.gunkel@krh.eu

F. von Ameln

Am Diekschloot 11b, 26506 Norden,

Deutschland

E-Mail: info@vonameln.net
} 
Moreno's theory must not become a cultural conserve that functions as prison for creativity“ (Kipper 1998, S. 115).

Moreno wäre sicherlich der Letzte gewesen, der die Entwicklung des Psychodramas hin zu einer solchen Kulturkonserve begrüßt hätte. Er war stets offen für die Auseinandersetzung mit anderen Verfahren (eindrucksvoll dokumentiert vor allem in „Psychodrama Vol. II“, Moreno 1975) und hat zahlreiche Artikel zur Kombination des Psychodramas mit anderen Methoden verfasst.

Das vorliegende Heft beleuchtet die Schnittstellen zwischen dem Psychodrama auf der einen Seite und anderen Verfahren und Konzepten auf der anderen Seite. Neben den Hauptbeiträgen, die mal von einer eher konzeptuellen, mal von einer eher praxisgeleiteten Perspektive aus geschrieben sind, enthält das Heft fünf Interviews mit Menschen, in deren Biografie das Psychodrama eine wichtige Rolle spielt, die sich dann aber auch anderen Verfahren zugewandt haben.

In der Debatte um integrative Arbeit ist immer wieder auf die Gefahr des Eklektizismus, d. h. die Kombination von methodischen Bestandteilen aus unterschiedlichen Verfahren ohne einen integrierenden theoretischen Rahmen hingewiesen worden. Zwar schneidet ein eklektisches Vorgehen empirisch nicht schlecht ab. Auch auf konkurrierende Theoriebestände Bezug zu nehmen, ist keineswegs als unprofessionell, sondern eher als adäquater Umgang mit der Komplexität des Gegenstands anzusehen: Die den verschiedenen Verfahren zugrundeliegenden Menschenbilder und Interpretationsfolien (Ätiologien etc.) können keinen Alleingeltungsanspruch erheben, sondern stellen theoretische Ordner dar, die stets nur einen Ausschnitt der Wirklichkeit beschreiben (Ameln et al. 2004, S. 271).

Nichtsdestoweniger gehört zum Professionalitätsverständnis jeder Beratung und Psychotherapie die Forderung, das eigene Handeln entsprechend der Professionsstandards des jeweiligen Formates begründen zu können. Beliebigkeit im Sinne eines ,anything goes“ kann daher - wenngleich möglicherweise in der Praxis durchaus erfolgversprechend - professionstheoretisch nicht das Ziel einer integrativen Arbeit sein.

In diesem Sinne sind verschiedentlich Versuche unternommen worden, ein Integrationsmodell zu erarbeiten, das die unterschiedlichen Verfahren in einen gemeinsamen Begründungszusammenhang stellt. Der wohl prominenteste Beitrag ist dabei das von Grawe entwickelte Konzept einer Allgemeinen Psychotherapie (Grawe et al. 2001). Grawe und seine Arbeitsgruppe vertraten die These, „Integration sei nicht vor allem auf der Ebene von Ansätzen, sondern auf der Ebene des Handelns in der einzelnen Therapie zu leisten“ (Caspar 2010, S. 18). In diesem Sinne könne sich das konkrete Vorgehen in einem therapeutischen Prozess (Analoges ließe sich für Beratung und vergleichbare Formate postulieren) nicht allein aus den praxeologischen Regeln eines Verfahrens ableiten, sondern müsse eine Vielzahl von Aspekten berücksichtigen, darunter die Probleme und Ressourcen der KlientInnen, die therapeutische Beziehung, Alltags-, Grundlagen und Veränderungswissen, allgemeine und störungsspezifische ätiologische Konzepte etc.

Dabei seien vier allgemeine Wirkprinzipien zu berücksichtigen, die Grawe aus zahlreichen Evaluationen herausdestilliert hat und die einen großen Teil der empirisch festgestellten Wirkungen von Psychotherapie erklären: 
1. Ressourcenaktivierung (Konzentration auf die Stärken und Fähigkeiten des KlientInnen und ihre Nutzbarmachung für den therapeutischen Prozess; insbesondere die Nutzung der therapeutischen Beziehung als Ressource).

2. Problemaktualisierung (die Probleme der KlientInnen im therapeutischen Sinne erfahrbar machen: „Was verändert werden soll, muss in der Therapie real erlebt werden. Oder: ,Reden ist Silber, real erfahren ist Gold““, Grawe 1995, S. 136).

3. Klärungsperspektive (Re- und Neukonstruktion der Bedeutungen, in denen sich der Patient im Verhältnis zu sich selbst und zu seiner Umwelt erfährt).

4. Bewältigungsperspektive (konkrete Hilfestellungen zur unmittelbaren Veränderung einer problematischen Lage oder eines problematischen Verhaltens der KlientInnen, z. B. in Form von Trainingsprogrammen).

Das Psychodrama hat mit seinen Konzepten der Begegnung sowie der Spontaneität und Kreativität (Ressourcenaktivierung), seiner umfangreichen Methodik zur Erforschung der Wirklichkeit der KlientInnen (Problemaktualisierung) oder den Möglichkeiten des Rollentrainings (Bewältigungsperspektive) auf allen diesen Dimensionen einiges zu bieten.

Grawe war der Überzeugung, dass die Integration von Methoden aus verschiedenen psychotherapeutischen Verfahren auf relevanten Fakten (insbesondere empirischer Evidenz, aber auch Praktikerwissen) beruhen müsse. Grawes Bestreben um Evidenzbasierung als Selektionskriterium ist nachdrücklich kritisiert worden (Tschuschke und Freyberger 2014), mit dem Argument, dass die Psychotherapieforschung bislang zwar immer wieder Evidenz für unspezifische Wirkfaktoren gefunden, aber verfahrensspezifische Wirkfaktoren niemals überzeugend belegt hat. Auch ist die Frage, inwieweit Ergebnisse aus randomisierten kontrollierten Studien (RCTStudien) überhaupt valide Rückschlüsse auf die Praxis zulassen.

Insgesamt zeichnet sich also ab, dass integrative Arbeit auf der Ebene einzelner Psychotherapie- oder Beratungsprozesse vor dem Hintergrund dieser Diskurse, die hier nur in aller Kürze angeschnitten werden können, durchaus geboten und hilfreich sein kann. Dies muss jedoch auf der Basis eines reflektierten Begründungsrahmens geschehen, der die (eher unspezifischen) von Grawe postulierten Wirkfaktoren sowie jeweils kontextspezifischen Faktoren wie z. B. die Ressourcen der KlientInnen oder persönliche Stärken und Schwächen des Therapeuten oder der Therapeutin berücksichtigt (vgl. Caspar 2010). Diese Position formuliert auch die gemeinsame Grundüberzeugung, die sich in vielen Beiträgen des vorliegenden Heftes ausdrückt.

Das Heft wird von einem Interview mit einem der intimsten Kenner des Psychodramas eröffnet, der aber heute vor allem für seine „Integrative Therapie“ steht. Hilarion Petzold beschreibt, wie er durch verschiedene biografische Begegnungen mit dem Psychodrama und anderen therapeutischen Verfahren in Kontakt kam. Petzold lotet die Parallelen zwischen diesen Verfahren aus und arbeitet vor diesem Hintergrund das Psychodrama als integratives Verfahren mit breit angelegtem Menschenbild, hoher theoretischer Reichweite und umfassenden behandlungstechnischen Möglichkeiten heraus.

Ulf Klein rekonstruiert in seinem Beitrag das Psychodrama als genuin systemisches Verfahren. Im ersten Teil zeigt er die Parallelen in Menschenbild, Beziehungsorien- 
tierung und Veränderungsverständnis auf, bevor er im zweiten Teil Berührungspunkte und Unterschiede in den methodischen Zugängen beider Verfahren herausarbeitet.

Auch Jan Bleckwedel arbeitet mit Kombinationen psychodramatischer und systemischer Zugänge, die er im nachfolgenden Interview schildert. Seine Konzeption für eine integrative Arbeit ist vor allem als kreative Kooperation zwischen BeraterInnen und KlientInnensystem angelegt, in der ein Verhandlungsprozess über den Einsatz konkreter Methoden entscheidet.

Matthias Lauterbach setzt systemische Methoden wie z. B. systemische Strukturaufstellungen gemeinsam mit psychodramatischen Elementen (etwa dem sozialen Atom oder Zeitlinien) im Rahmen seines Gesundheitscoachings ein. Lauterbach entwickelt zunächst - vor allem mit Bezug auf Dürckheim - ein mit dem Psychodrama kompatibles Verständnis von Gesundheit als Erleben von Sinnhaftigkeit und Stimmigkeit, bevor das konkrete Vorgehen geschildert und an einem Fallbeispiel illustriert wird.

Roger Schaller befasst sich in seinem Beitrag mit der Frage, was Psychodrama und Verhaltenstherapie voneinander lernen können. Während PsychodramatikerInnen, so seine These, etwa vom verhaltenstherapeutischen Training sozialer Fertigkeiten profitieren können, sieht Schaller im Konzept des Psychodramas als globale Werkstätte (Baars) und als Externalisierung des Mentalisierens der KlientInnen Potenzial für neuere verhaltenstherapeutische Ansätze.

Das folgende Interview mit Christa Rohde-Dachser leitet über zur Auseinandersetzung mit psychoanalytischen und tiefenpsychologischen Ansätzen. Ausgehend von ihrem sehr persönlichen Zugang zum Psychodrama schildert die Autorin vor allem, wie sie bei der Schilderung der KlientInnen aufsteigende innere Szenen der Therapeutin in szenische Arbeit auf der Bühne übersetzt.

Volker Riegels überprüft in seinem Artikel die Frage, ob es auf theoretischer Ebene oder auf der Ebene der Behandlungstechnik Potenzial für eine Integration von Psychodrama und Psychoanalyse bzw. tiefenpsychologisch fundierter Psychotherapie geben kann. zur Beantwortung dieser Frage zieht er den Intersubjektivismus sowie die Control-Mastery-Theorie heran und veranschaulicht seine Überlegungen durch ein abschließendes Fallbeispiel.

Ebenso wie Christa Rohde-Dachser gehört auch Ralf Binswanger zu den Pionieren des Psychodramas in Europa, die sich später der Psychoanalyse zuwandten. Er spricht über die Faszination, die das Psychodrama auf ihn ausübte und über Erkenntnisse, die die Psychoanalyse vom Psychodrama übernehmen könnte, kommt hinsichtlich der Integration beider Verfahren aber zu einem eher skeptischen Fazit.

Birgit Koerdt-Bruening stellt in ihrem Beitrag zunächst die Katathym Imaginative Psychotherapie vor und schildert dann anhand einer Fallvignette, wie dieses Verfahren mit psychodramatischen Elementen kombiniert werden kann. Dabei setzt sie vor allem den Rollentausch - zunächst in der Imagination, dann auf der Psychodrama-Bühne - ein.

Albrecht Boeckh setzt Psychodrama und Gestalttherapie in theoretischer wie methodischer Hinsicht miteinander in Beziehung. Seine Hauptthese lautet, dass sich beide Verfahren angesichts ihrer unterschiedlichen Foci gut ergänzen können, weil die Gestalttherapie stärker auf die individuelle Entwicklung, das Psychodrama dagegen stärker auf die Entwicklung der sozialen Beziehungen fokussiert. 
Björn Migges Beitrag ist ein Plädoyer für eine stärkere Beachtung des Psychodramas im Rahmen eines integrativen Coaching-Konzepts. Ausgehend von einer Darstellung der Kernkompetenzen, über die ein Coach verfügen sollte, zeigt er, wie das Psychodrama in der Coaching-Ausbildung zur Förderung dieser Kernkompetenzen eingesetzt werden kann. Abschließend stellt Migge Überlegungen an, warum das Psychodrama in der Coaching-Szene bislang recht wenig bekannt ist und plädiert für eine stärkere Profilierung des Psychodramas in diesem Bereich.

PsychodramatikerInnen werden die Arbeit von Friedemann Schulz von Thun als vertraut empfinden. Wie das nachfolgende Interview zeigt, ist diese Verbindung kein Zufall: Schulz von Thun und sein Kollege Karl Benien berichten über psychodramatische Inspirationen für ihre Arbeit und zeigen anhand von Beispielen, wie sie psychodramatische Elemente in Vorträgen und Seminaren einsetzen.

Jutta Heppekausen stellt anhand von Beispielen aus der Praxis dar, wie der Ansatz der Gewaltfreien Kommunikation in Supervisionsprozessen mit Psychodrama-Elementen kombiniert werden kann. So können vor allem nicht bewusste Erlebensanteile in Konflikten zugänglich gemacht werden, um auf dieser Basis reflektiertere Entscheidungen treffen zu können.

Der nachfolgende Beitrag greift das Thema der integrativen Arbeit mit dem Psychodrama aus einer anderen Perspektive auf: Das multidisziplinäre Team der Wiener Beratungsstelle ,Frauen beraten Frauen“ zeigt, wie die psychodramatische Arbeit mit verschiedenen Unterstützungsangeboten kombiniert und gebündelt werden kann, um Hilfestellung in komplexen Problemlagen zu bieten.

In der Rubrik „Der andere Artikel“ schildert Katharina Witte in einem sehr anschaulichen Beitrag, wie das Playbacktheater als Methode zur Unterstützung von Veränderungsprozessen eingesetzt werden kann.

Neben den weiteren Rubriken wie Rezensionen oder Veranstaltungsterminen sei besonders auf den Vernetzungs-Beitrag von Stefan Flegelskamp hingewiesen, der in trauriger Aktualität - zeigt, wie Psychodrama-Arbeit dazu beitragen kann, traumatisierten Kindern im Gazastreifen zu helfen.

Wir wünschen allen LeserInnen eine anregende und erkenntnisreiche Lektüre.

\section{Literatur}

Ameln, F. v. \& Wieser, M. (Hrsg.) (2014). Jacob Levy Moreno revisited - ein schöpferisches Leben. Zum 125. Geburtstag. Wiesbaden: Springer VS.

Ameln, F. v., Gerstmann, R. \& Kramer, J. (2004). Psychodrama. Berlin: Springer.

Caspar, F. (2010). Wie allgemein ist Grawes „Allgemeine Psychotherapie“? Psychotherapie im Dialog, 11(1), 15-21.

Grawe, K. (1995). Grundriß einer Allgemeinen Psychotherapie. Psychotherapeut, 40, 130-145.

Grawe, K., Donati, R. \& Bernauer, F. (2001). Psychotherapie im Wandel: von der Konfession zur Profession (5. Aufl.). Göttingen: Hogrefe.

Kipper, D. A. (1998). Psychodrama and trauma: Implications for future interventions of psychodramatic role-playing modalities. International Journal of Action Methods - Psychodrama, Skill Training, and Role Playing, 51(3), 113-121.

Moreno, J.L. (1975). Psychodrama (Vol. II, Second Edition). Beacon: Beacon House.

Tschuschke, V. \& Freyberger, H. J. (2014). Zur aktuellen Situation der Psychotherapiewissenschaft und ihrer Auswirkungen - eine polemische Analyse der Fakten. (Unveröff. Manuskript). 